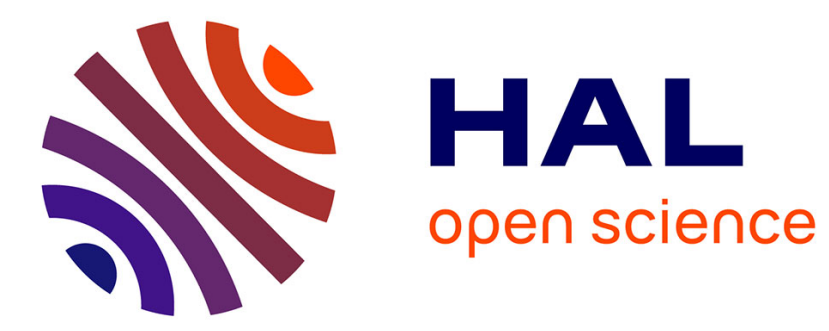

\title{
The Virgo interferometric gravitational antenna
}

F. Acernese, P. Amico, M. Al-Shourbagy, S. Aoudia, S. Avino, D. Babusci, G.

Ballardin, R. Barillé, F. Barone, L. Barsotti, et al.

\section{To cite this version:}

F. Acernese, P. Amico, M. Al-Shourbagy, S. Aoudia, S. Avino, et al.. The Virgo interferometric gravitational antenna. Optical Diagnostics and Monitoring (OPTIDIMON), Mar 2004, Bacoli (Napoli), Italy. pp.1-16. in2p3-00024243

\section{HAL Id: in2p3-00024243 https://hal.in2p3.fr/in2p3-00024243}

Submitted on 24 Mar 2006

HAL is a multi-disciplinary open access archive for the deposit and dissemination of scientific research documents, whether they are published or not. The documents may come from teaching and research institutions in France or abroad, or from public or private research centers.
L'archive ouverte pluridisciplinaire HAL, est destinée au dépôt et à la diffusion de documents scientifiques de niveau recherche, publiés ou non, émanant des établissements d'enseignement et de recherche français ou étrangers, des laboratoires publics ou privés. 


\section{The V irgo interferom etric gravitational antenna}

F.A cemesel , P.Am ico ${ }^{\mathrm{x}}$,M .A l-Shourbagy ${ }^{\mathrm{xi}}$, S.A oudia** , S.Avindll,

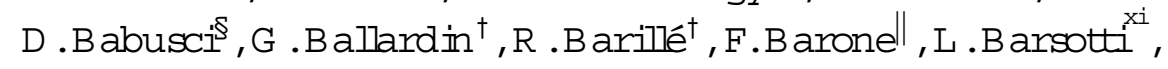
M . Barsuglia ${ }^{\dagger \dagger}$, F.Beauville*,$M$ A.B izouard ${ }^{\dagger \dagger}, C$. Boccara ${ }^{\ddagger \neq}$, F.Bondu**, L .Bosi , C .Bradaschia ${ }^{x}$, S.B raccini ${ }^{x i}$,A . B rillet** , V.Brisson $^{\dagger \dagger}$, L . B rocco ${ }^{\text {xii }}$, D .Buskulic* , E .C allonil , E .C am pagna ${ }^{\ddagger}$, F.C avalier ${ }^{\dagger \dagger}$, R .C avalieri ${ }^{\dagger}$, G .C ella ${ }^{x i}, E$.C hassande-M ottin** , C . C Orda $^{\text {xi }}$, A .C .C lapson ${ }^{\dagger \dagger}$, F.C leva** , J.P.C Coulon** , E .C uoco ${ }^{\dagger}$,

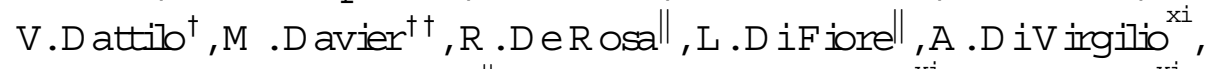
B .D ujardin**,A .E leuteril , D .Enard ${ }^{\dagger}$, I. Ferrante ${ }^{\text {xi }}$,F.F idecaro ${ }^{x i}$, I.Fiori , R .Flam inio* , J.D .Foumier** , S.Frasca 'xii ,F.Frasconi ${ }^{\dagger}$, A .Freise ${ }^{\dagger}$, L .G am m aitoni $i^{x}$, A .G ennai , A .G iazotto ${ }^{x i}, G$.G iordano ${ }^{\S}$, L .G iordanoll , R .G ouaty* , D .G rosjean* , G .G uidi , S.H ebri ${ }^{\dagger}$, $\mathrm{H} . \mathrm{H}$ eitm ann** , P.H ello ${ }^{\dagger \dagger}, \mathrm{L} . \mathrm{H}$ ollow ay $^{\dagger}$, S . K reckelbergh ${ }^{\dagger \dagger}$, P.La Penna ${ }^{\dagger}$, V.L Loriette ${ }^{\ddagger}, \mathrm{M}$.Loupias ${ }^{\dagger}, \mathrm{G}$.Losurdo ${ }^{\ddagger}$, J.M .M ackow skil, E.M ajorana , C.N .M an**,M .M antovani ${ }^{\text {xi }}$, F.M archesoni ${ }^{x}$, F.M arion* , J.M arque ${ }^{\dagger}$, F.M artelli ${ }^{\ddagger}$, A .M asserot*, M .M azzoni $i^{\ddagger}$, L .M iland , C .M oins ${ }^{\dagger}, \mathrm{J} . \mathrm{M}$ oreau ${ }^{\ddagger \dagger}, \mathrm{N} . \mathrm{M}$ orgado", B .M ours* ,A.Pai , C .Palom ba ${ }^{\text {xii }}$, F.Paoletti ${ }^{\dagger}$, S.Pardill, A .Pasqualetti ${ }^{\dagger}$, R . Passaquieti ${ }^{x i}$, D .Passuello ${ }^{x i}$, B .Pemiola ${ }^{\ddagger}$, F.P iergiovanni L .P inard ${ }^{\ddagger} R$.Poggiani ${ }^{x i}, M$.Punturo ${ }^{x}, P . P u p p o{ }^{x i i}$,

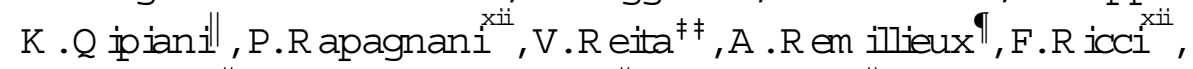
I.R icciardil , P.R uggi ${ }^{\dagger}$, G .Russoll , S.Solim end , A .Spallicci**, R . Stanga ${ }^{\ddagger}, \mathrm{R}$. Tadde $i^{\dagger}, \mathrm{D}$. Tom bolato*, $\mathrm{M}$. Tonelli $\mathrm{x}^{\mathrm{xi}}, \mathrm{A}$. Toncelli $i^{\mathrm{xi}}$, E .Toumefier* , F.Travasso , G .Vajente ${ }^{x i}$, D .Verkindt* , F.Vetrano ${ }^{\ddagger}$, A .V iceré ${ }^{\ddagger}$, J.Y. .V inet** ,H .Vocca ${ }^{*}, M$.Y vert* and $Z$ Z Zhang ${ }^{\dagger}$

* Laboratoire d'Annecy-le-Vieux de Physique des Particules, Annecy-le-Vieux, France;

${ }^{\dagger}$ European G ravitational o bservatory (EG O ), Cascina (P i) , Italia;

${ }^{\ddagger} \mathbb{N}$ FN , Sezione di Firenzet roino, Sesto Fiorentino, and/or U niversità di Firenze, and/or U niversità diU ubino, Tralia;

$\S_{\mathbb{N} F N}$, LaboratoriN azionalidiFrascati, Frascati (Rm ), Italia;

ILM A, Villeurbanne, Lyon, France;

$\|_{\mathbb{N} F N}$, sezione diN apoliand/or U niversità diN apoli "Federico II" Complesso U niversitario diM onte S Angelo, and/or U niversità disalemo, Fisciano (Sa), Italia;

** D epartem entArtem is - O bservatoire de la Côte d'A zur, BP 4220906304 


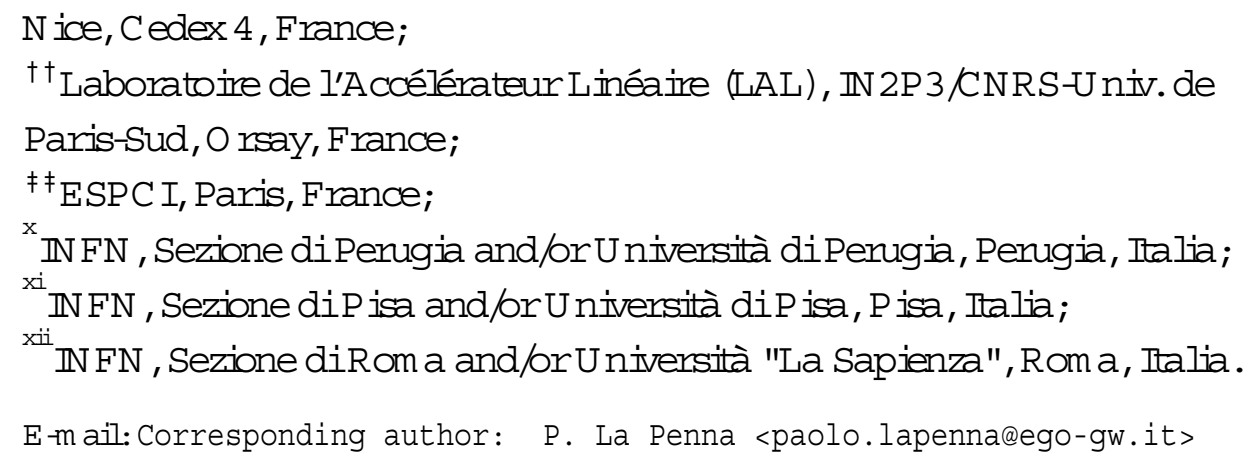

A bstract. The interferom etric gravitational w ave detectors represent the ultim ate evolution of the classical M ichelson interferom eter. In order to m easure the signal produced by the passage of a gravitational wave, they aim to reach unprecedent sensitivities in m easuring the relative displacem ents of the $\mathrm{m}$ inors. O ne of them, the $3 \mathrm{~km}$-long Virgo gravitational w ave antenna, which w illbe particularly sensitive in the low frequency range $(10-100 \mathrm{H} \mathrm{z})$, is presently in its com $\mathrm{m}$ issioning phase. In this paper the various techniques developed in order to reach its targetextrem e perform ance are outlined.

Subm itted to: C lass. Q uantum G rav.

PA CS num bers: $04.80 \mathrm{Nn}, 95.55 . \mathrm{Ym}$

\section{Introduction}

The present ground-based interferom etrical gravitational w ave detectors V irgo [2], L IG O [3], TAM A 300 [4], GEO 600 [5]) aim to reach a spectral strain sensitivity of $h$ about $10^{-23} / \sqrt{\mathrm{H} \mathrm{z}}-10^{-22} / \sqrt{\mathrm{H} \mathrm{z}}$ in a frequency range around $100 \mathrm{~Hz}$. This means, in term $\mathrm{s}$ of relative displacem ents of the test $m$ asses, to measure length varitions of less than $10^{-19} \mathrm{~m} / \sqrt{\mathrm{Hz}}$ (see figure 1 ). In order to reach this extrem e sensitivity, special optical configurations have been developed.

The passage of a gravitational w ave can be detected in the output interferom etric signal as a relative displacem entof a setof quasi-free falling $m$ asses (suspended $m$ inors). V irgo, $m$ ainly consisting of a $3 \mathrm{~km}$-long $\mathrm{M}$ ichelson interferom eter, $\mathrm{w}$ ith Fabry-Perotcavities in the arm sand pow er recycling, shares w ith other experim ents, such as LIG O [3], TA M A [4] and G EO [5], substantially the sam e optical detection principle, and it aim s to detect gravitational w aves em itted by astrophysical sources [2] in a frequency range betw een a few $\mathrm{H} z$ and a few $\mathrm{kH} z$. In particular, V irgo, thanks to its peculiar attenuating system providing the highest passive isolation perform ance, $w$ ill be $m$ ore sensitive than the other detectors at low frequency (10$100 \mathrm{H} z$ range) , aim ing ata displacem ent sensitivity of $10^{-17} \mathrm{~m} / \sqrt{\mathrm{H}} \mathrm{z}$ at $10 \mathrm{H} \mathrm{z}$ and at the level $10^{-19} \mathrm{~m} / \sqrt{\mathrm{H} \mathrm{z}}$ atabout $100 \mathrm{H} \mathrm{z}$. In term s of fringe sensitivity, the progress $\mathrm{w}$ ith respect to the $M$ ichelson and M orley experim entw ould be from their $1 / 100$ [1] to the present $1 / 10^{12}$ of the fringe (but the frequency reange atw hich the m easurem ent is perform ed is different). 


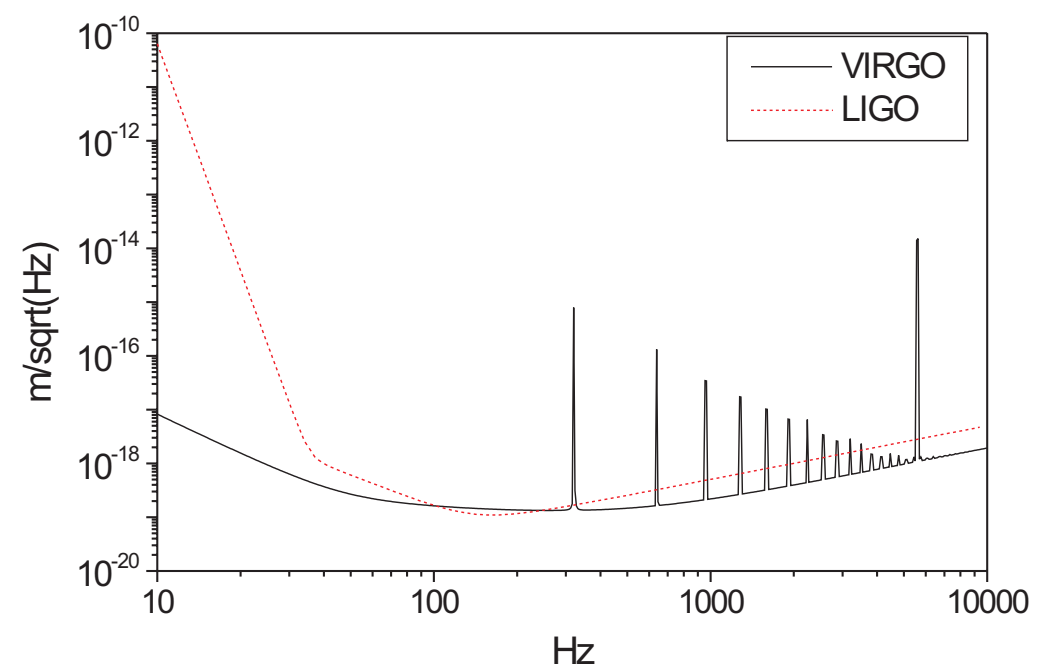

F igure 1. Com parison of the design displacem entsensitivity of the tw o interferom eters $V$ irgo and $\mathrm{LIG} O$, expressed in $\mathrm{m} / \sqrt{\mathrm{H} z}$, as a function of the frequency.

2. Basic principles of operation of an interferom etric antenna

The first idea of the interferom etric detection of gravitational waves rem ounts up to the 60 's [6]. The passage of a gravitational w ave, com ing from a direction perpendicular to the line connecting tw $\mathrm{m}$ inors, suspended as quasi-free test $m$ asses at a certain distance $\mathrm{L}$ one from the other, $w$ ill be seen by a laser lightbeam travelling along this line as a displacem ent of the sam e test m asses. The result $\mathrm{w}$ ill be a change in the interference condition betw een the beam s com ing from the tw o opposite directions. Since the action of the gravitational w ave on a length $\mathrm{L}$ is essentially described by its adim ensional strain $\mathrm{h}=\Delta \mathrm{L} / \mathrm{L}$, the quantity $\Delta \mathrm{L}$ to be $\mathrm{m}$ easured is enhanced if the length $\mathrm{L}$ is larger. $\mathrm{G}$ iven the expected values for an adim ensional strain of astrophysical source (about $10^{-23} / \sqrt{\mathrm{H} \mathrm{z}}-10^{-22} / \sqrt{\mathrm{H} \mathrm{z}}$ in the 10-100 $\mathrm{H} \mathrm{z}$ frequency range), the $\Delta \mathrm{L}$ to be m easured is extrem ely $\mathrm{sm}$ all. For this reason the ground based gravitational w ave interferom eters are as long as possible, of the order of several km ( $V$ irgo is $3 \mathrm{~km}$ long, LIG O is $4 \mathrm{~km}$ long).

\subsection{Opticalcavities}

In order to increase the optical path, the firstpossibility w ould be to use delay lines: the light could be $\mathrm{m}$ ade travel $\mathrm{m}$ any tim es inside tw $\mathrm{m}$ inors spaced several $\mathrm{km}$ apart. This device is too difficult to control, thus the use of optical cavities (Fabry-Perot) has been preferred. In a Fabry-Perot cavity having finesse $\mathrm{F}$ the dephasing of the reflected light is enhanced by a factor $2 / \pi \times F$. Therefore, $w$ ith a finesse $F=50$, a cavity $3 \mathrm{~km}$-long can be seen as optically equivalent to an about $120 \mathrm{~km}$-long one. 


\section{2. M ichelson configuration}

In principle a single cavity could be used. H ow ever, the signal com ing from a single cavity w ould be dom inated by the laser frequency noise $\Delta v$ (typically of the orderof $10^{4} \mathrm{H} \mathrm{z} /(\mathrm{f} \sqrt{\mathrm{H} \mathrm{z}}$ ) . For a cavity having length $\mathrm{L}$ of the order of $1 \mathrm{~km}$, and a laser frequency $v=2.8 \times 10^{14}$, the equivalent displacem ent noise w ould be $\Delta \mathrm{L}=\Delta \mathrm{v} / \mathrm{v} \times \mathrm{L} \cong 3 \times 10^{-10} \mathrm{H} \mathrm{z} / \sqrt{\mathrm{H} \mathrm{z}}$ at $100 \mathrm{~Hz}$. In order to overcom e this Im it the interferom eter configuration is used: in an idealM ichelson, having no arm s asym m etry, there is no frequency noise injection in the interference betw een the beam s com ing from the tw o arm $\mathrm{s}$. This is the reason because all the gravitational antennas have the $\mathrm{M}$ ichelson interferom eter configuration. In a gravitational w ave intenferom etric antenna high-quality optics are suspended to act as quasi-free test $m$ asses at the end of the M ichelson interferom eter arm s, the arm s being, in V irgo, LIG O and TA M A, optical cavities.

\section{3. D ark fringe}

U sing laser beam $S$, the first fundam ental noise source is the shot noise connected to the corpuscularnature of the light. In order to im prove the shotnoise signal-to-noise ratio, itcan be show $n$ that the shotnoise signal to noise ration is $m$ axim ized if the intenferom eter is kept on the dark fringe, that is the tw o beam s com ing from the tw 0 arm $s$ are m ade destructively interfering. A ll the light is therefore reflected back tow ards the laser source. In this w ay, the w hole interferom eter behaves like a m inror, reflecting back all the incom ing light. Since the shotnoise signal-to-noise ratio im proves $w$ ith the square rootof the pow er, it is convenient to increase as m uch as possible the light stored inside the interferom eter.

\subsection{Pow er recycling}

O nce the lim it in the increase of the incom ing laserpow er is reached, a further im provem ent can be obtained by reflecting again, tow ards the intenferom eter, the light com ing back to the laser. For this reason, betw een the laser and the interferom eterbeam splitter, anotherm inor, called the recycling $\mathrm{m}$ inor, is placed, which, together $\mathrm{w}$ ith the interferom eter itself, form s a furtheroptical cavity in w hich the light is stored. The lightpow er inside the recycling cavity, i.e. the light im pinging onto the intenferom eter beam splitter, is enhanced by a "recycling" factor.

\subsection{H eterodyne detection}

A further lim it to the sensitivity are the pow er fluctuations of the laser light. The presently available noise source are not shot noise dom inated in the frequency of interest forgravitational w ave detection $(10 \mathrm{H} \mathrm{z}-10 \mathrm{kH} \mathrm{z})$. O ne trick to overcom e the pow erfluctuation problem is to shift the detection frequency in a frequency range w here the laserbeam is shot noise dom inated. For this reason the laser light ism odulated in phase ata frequency of several M H z, where the laser is shot noise dom inated, before entering the intenferom eter, and then dem odulated by the sam e reference, in the standard Pound-D reverH all (PD H ) schem e [7] [8] . At the dark fringe output of the interferom eter the beating betw een the camier and the 
sidebands is detected (heterodyne detection), when the destructive interference condition is m odified by the passage of a gravitational $w$ ave. In order to have an available signal, the sidebands have to be partially transm itted to the dark port: this is obtained by introducing a slight asym m etry in the arm s length, so that when the camier is on the perfect destructive interference the sidebands are partially tran.m itted (Schnupp's technique [9]).

The arm s asymmetry imposed by the Schnupp's technique, together w ith other arm s asymm etries (like cavity finesse asymm etries), have as a consequence that part of the frequency noise of the laser is reintroduced in the interferom eter. This im poses to stabilize the laser frequency, up to a level where its effect is low er than the shotnoise (the frequency stabilization schem e adopted in V irgo w illbe described in 4.3.

\subsection{Autom atic alignm ent}

In addition to the interferom eter is longitudinal locking, an angular control system is needed to $\mathrm{m}$ aintain the $\mathrm{m}$ inors in an aligned position $\mathrm{w}$ ith respect to one another and the incom ing beam . The alignm ent system is not referred to the ground, it rather keeps the interferom eter autom atically aligned on the incom ing beam . Several techniques, know n asw avefrontsensing techniques [10] are used in the gravitational w ave interferom eters: they all take advantage, analogously to the Pound-D rever $\mathrm{H}$ all technique, of a high frequency $(\mathrm{M} \mathrm{H} \mathrm{z})$ m odulationdem odulation technique. The autom atic alignm ent schem e designed for Virgo [12] uses the Anderson technique [11]. Them odulation frequency is chosen so that the firstordertransverse m odes ( $T \mathrm{EM}_{01}$ ) of the sidebands are resonant in the arm cavities. O nce all the cavities of the interferom eter are locked at their resonance, the transm itted light is detected by differential w ave-front sensors, producing photo currents w hich are dem odulated and then opportunely $\mathrm{m}$ ixed to achieve signals proportional to the $\mathrm{m}$ isalignm ents betw een the opticalcom ponents. These signals are then filtered and sentby feedback to them inors at the levelof them arionette $\mathrm{w}$ ith a controlbandw idth of a few $\mathrm{H} z$, so thatnoise is not reintroduced in the detection band.

\subsection{Locking}

In order to attain the desired sensitivity, the interferom eterhas to be placed and kept in a specified w orking point, i.e. $\mathrm{w}$ ith the arm s at the optical resonance of the cavities, the M ichelson on the outputdark fringe and the recycling cavity atits resonance. Thism eans that the various lengths and positions of the m imors have to be actively controlled, this operation being called longitudinal locking of the interferom eter.

\section{The V irgo interferom eter}

\subsection{Virgo general layout}

The optical layoutofV irgo is show $\mathrm{n}$ in figure 2 : a laserbeam (20W @ 1064nm) isproduced by a d : $\mathrm{YVO}_{4}$ high pow er laser injection, locked to a $1 \mathrm{~W}$ N d :YAG m aster laser. The laser light 
is m odulated in phase at a frequency of $626 \mathrm{M} \mathrm{H} \mathrm{z}$ before entering the vacuum system at the injection bench $(\mathbb{B})$, and is then pre-stabilized by the $144 \mathrm{~m}$ long inputm ode-cleaner $(\mathbb{M} C)$, to a few tens of $\mathrm{kH} \mathrm{z}$ using a standard Pound-D rever $\mathrm{H}$ all (PDH) schem e [7] [8]. The low frequency pre-stabilization is perform ed by actively controlling the length of the $\mathbb{I M} C$ to lock the laser frequency to the length of a $30 \mathrm{~cm}$ m onolithic triangular cavity (RFC) suspended in vacuum . A $10 \mathrm{~W}$ pow erbeam em itted from the injection system enters into the interferom eter (TFF) through the pow er-recycling $m$ inor (PR). The beam is split at the level of the beam splitterm inor (BS), and enters the tw o 3km long Fabry-Perotcavities (north cavity and west cavity).

Together w ith the PR m inor, the M ichelson ITF form s a Febry-Perot cavity, the power recycling cavity, w ith an optical recycling gain of $50 \mathrm{w}$ hen the ITF is at its working point. In this state, the expected pow er upon the BS is $500 \mathrm{~W}$. The $3 \mathrm{~km}$ Fabry-Perot cavities have a finesse of 50 (optical gain about 32) : the final pow ercirculating inside the $\mathrm{TF}$ is therefore estim ated to be around $8 \mathrm{~kW} . \mathrm{W}$ ith the ITF at the operating point, the gravitationalw ave signal is extracted on the dark portbeam, which passes through the outputm ode-cleaner (OM C ), to reach a set of 16 InG aA S photodiodes (B 1), by w hich the dark port signal is reconstructed. $O$ ther signals are extracted from the $\mathbb{T F}$, essentially for control purposes: figure 2 show $\mathrm{s}$ the benches detecting the beam s transm itted by the long Fabry-Perot cavities (NB and W B ), the beam reflected by the ITF (DT), and the beam reflected by the second face of the BS (DB ). In figure 3the noise sources lim iting the $V$ irgo sensitivity are show $\mathrm{n}$ : the $\mathrm{m}$ ain lim iting noise contributions w ill be seism ic disturbances below $4 \mathrm{H} \mathrm{z}$, therm al noise up to $100 \mathrm{~Hz}$ and shot noise athigher frequencies.

\section{Optics}

The V irgo otpical design im poses very large (diam eter $350 \mathrm{~m} \mathrm{~m}$ ) and heavy optics (20 kg)for the interferom eter suspended $\mathrm{m}$ inors and beam splitter. The total losses at $1064 \mathrm{~nm}$ of each $m$ irror (inchuding absorption, scattering and large-scale w avefront deform ation) should not exceed 100 parts perm illion (ppm). There are specific constraints on the absorption $(<5$ ppm ) due to the therm al lensing and on the scattering level $(<5$ ppm ) to $\mathrm{m}$ inim ize the noise on the interferom eter output, due to the scattered light. For these reasons $m$ inrors and beam splitters are super-polished piecesm ade of a new types of silica (Suprasil311 SV , Suprasil312 SV ), w th very low absorption and scattering, $m$ anufactured by the G erm an com pany H eraeus, developed in collaboration w ith ESPC I (Paris). The OH content is very low ( $<50$ ppm ), the refractive index is hom ogeneous in all directions and the birefiringence is very low $\quad<5$ 10-4 $\mathrm{rad} / \mathrm{cm}$ ). The bulk absorption of the silica substrates crossed by the V $\mathbb{R} G O$ laser beam has been $\mathrm{m}$ easured [13] as being less than $0.7 \mathrm{ppm} / \mathrm{cm}$. The flatness of these large com ponents is $8 \mathrm{~nm} \mathrm{RM} \mathrm{S}$ on $150 \mathrm{~mm}$. The lim iting factor of the wavefront flatness is the substrate: since the polishers can not guarantee every tim e better w avefronts, the substrate sunface is conrected before deposition by using a Conrective Coating technique [14]. The m inrors, after final coating w ith reflective quarter w avelength layers of Si 2 and Ta2O 5, exhibit a global RM S flatness of the order of $3 \mathrm{~nm}$ over $150 \mathrm{~m} \mathrm{~m}$, an average absorption of less than 1 ppm 


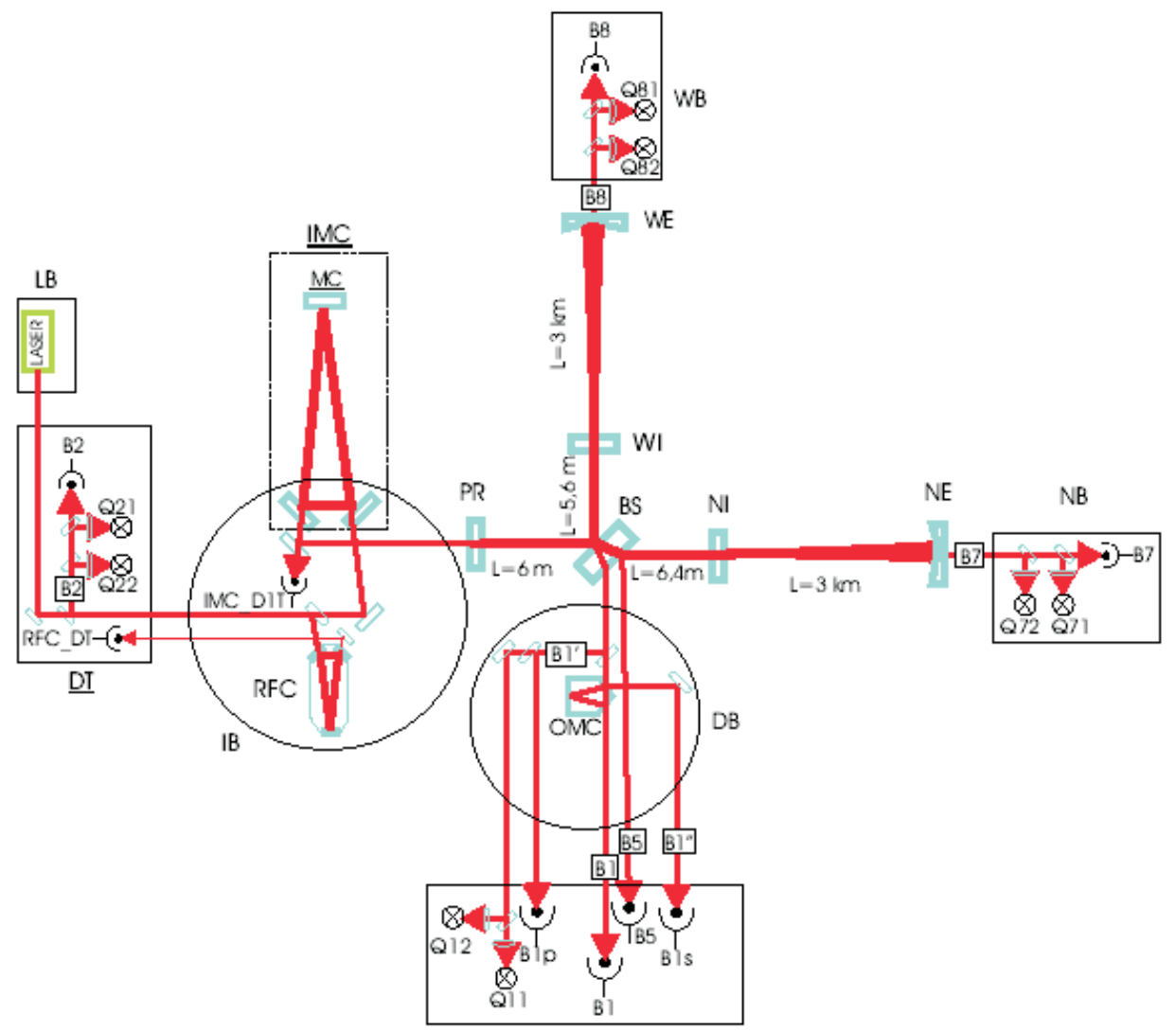

F igure 2.Virgo optical layout

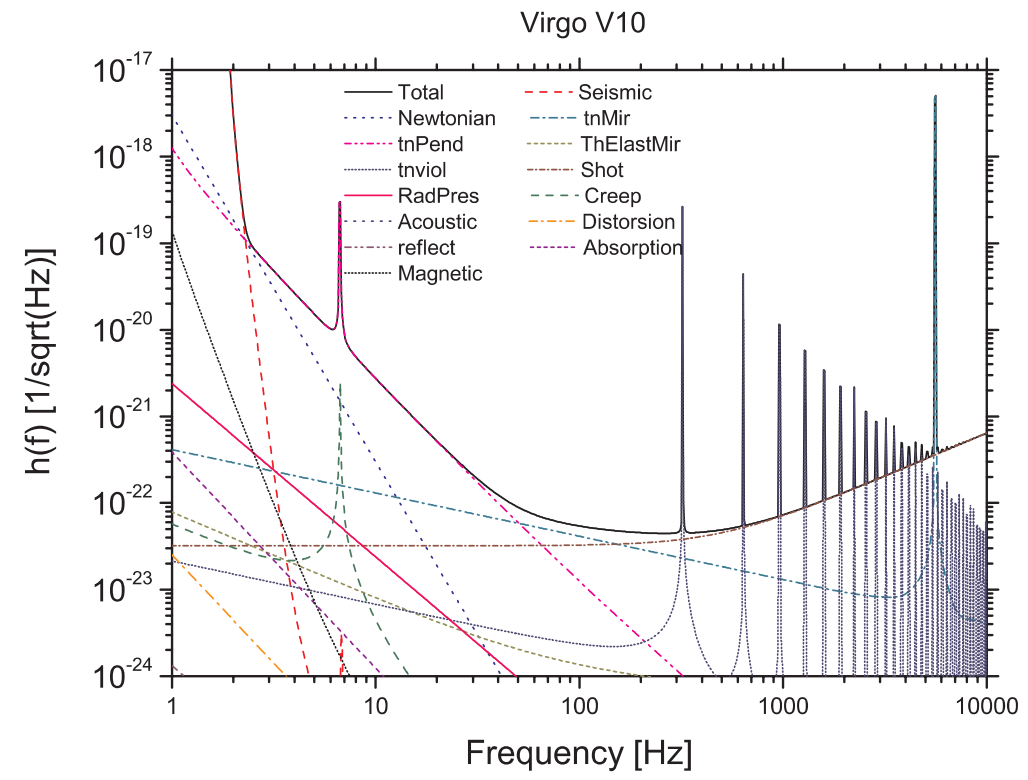

F igure 3. Virgo design sensitivity $\mathrm{w}$ ith the lim iting noise sources: seism ic noise up to $4 \mathrm{~Hz}$, therm alnoise up to $100 \mathrm{~Hz}$ and shotnoise athigher frequencies. 
and a scattering of the order of 5 ppm , thus being the m ostperform ing existing large optics.

\subsection{Suspensions}

In all the present ground based interferom eter the testm asses are m inors, isolated from the ground by suspending them to pendula. The V irgo seism ic isolation, being m ore com plex than those of the detectors, provides the highestpassive isolation perform ance. The testm asses are located in an ultra-high vacuum system (from $10^{-9} \mathrm{~m}$ bar forH 2 up to $10^{-14}$ forhydrocarbon) and suspended from a sophisticated seism ic isolation system the Superattenuator (SA ). The SA is a m ultistage, 10-m -tall, m ultipendularsuspension, w hich is effective in isolating the test $\mathrm{m}$ asses from the seism ic noise for frequency higher than a few $\mathrm{H} \mathrm{z}$ (the pendulum resonance frequency are all confined below a couple of $\mathrm{H} \mathrm{z}$ ). The seism ic attenuation system is either passive and active. The passive filtering is provided by the SA , a chain of m echanicalfilters. The first stage of the SA [15] is an inverted pendulum (IP) preisolating stage [16]. A chain of five m echanicalfilters is suspended from the top of the IP. From the last stage of the chain (the so called "filter 7" [17]) an anvil shaped steel stage, the so called "m arionette" [18], is suspended by a steelw ire. The payload, suspended from the m arionette, is form ed by the test $m$ ass and by an alum inum reference $m$ ass (RM), independently suspended behind the $m$ inor. The passive attenuation of the whole chain is better than $10^{-14}$ at $10 \mathrm{H} \mathrm{z}$, comresponding to an expected residual m inorm otion of $10^{-18} \mathrm{~m} / \sqrt{\mathrm{H}} \mathrm{z}$ at the sam e frequency. D ue to the residual low frequency $m$ otion of tens of $m$ icrons at the resonances of the SA, the frequencies of the whole chain norm alm odes ranging betw een $40 \mathrm{~m} \mathrm{~Hz}$ and $2 \mathrm{H} \mathrm{z} \mathrm{w}$ ith quality factors up to $10^{3}$, the SA is designed to allow an active control of the $m$ inrorposition overa very large dynam ic range. In order to allow lock acquisition, i.e. to confine the residual $m$ inror m otion below $1 \mu \mathrm{m}$, control forces are exerted on three actuation points: at the IP top stage level, perform ing an inertial active dam ping of the resonantm otions of the SA [19]; using the m arionette coils to steer the suspended $\mathrm{m}$ inror [18] $\mathrm{w}$ ith respect to the last stage of the chain; and directly on the $m$ inror, through coils supported by the RM which can act on four m agnets m ounted on the holder of the $m$ inor. A local control system referred to the ground, is active in the bottom part of each SA in order to keep the longitudinal displacem ent of the m inors below $1 \mu m$ Im s. It uses as a signal tw o laser beam s em itted by lasers leaning on ground outside each tow er. These beam s enter into the tow er through an optical w indow, one is reflected one by a $\mathrm{m}$ inor on the $\mathrm{m}$ arionetta and the other one by the suspended $\mathrm{m}$ inor itself, and then im pinge on respective PSD detectors, afterpassing through dedicated opticalsystem s, thus being used as optical levers for reconstructing the $m$ inorm ovem ents. A comection feedback using this signals allow s to dam $\mathrm{p}$ and control the local angularm otion of the m inors below 1prad RM S and $m$ akes the acquisition of the longitudinallock of the interferom eterpossible w ith a lim ited actuation force, thus preventing noise reintroduction in the detection band. Since this system is referred to the ground, and therefore lim ited by the seism ic noise, once the longitudinal locking of the intenferom eter is acquired the localcontrolsystem is sw itched off and replaced by the autom atic alignm entsystem . 


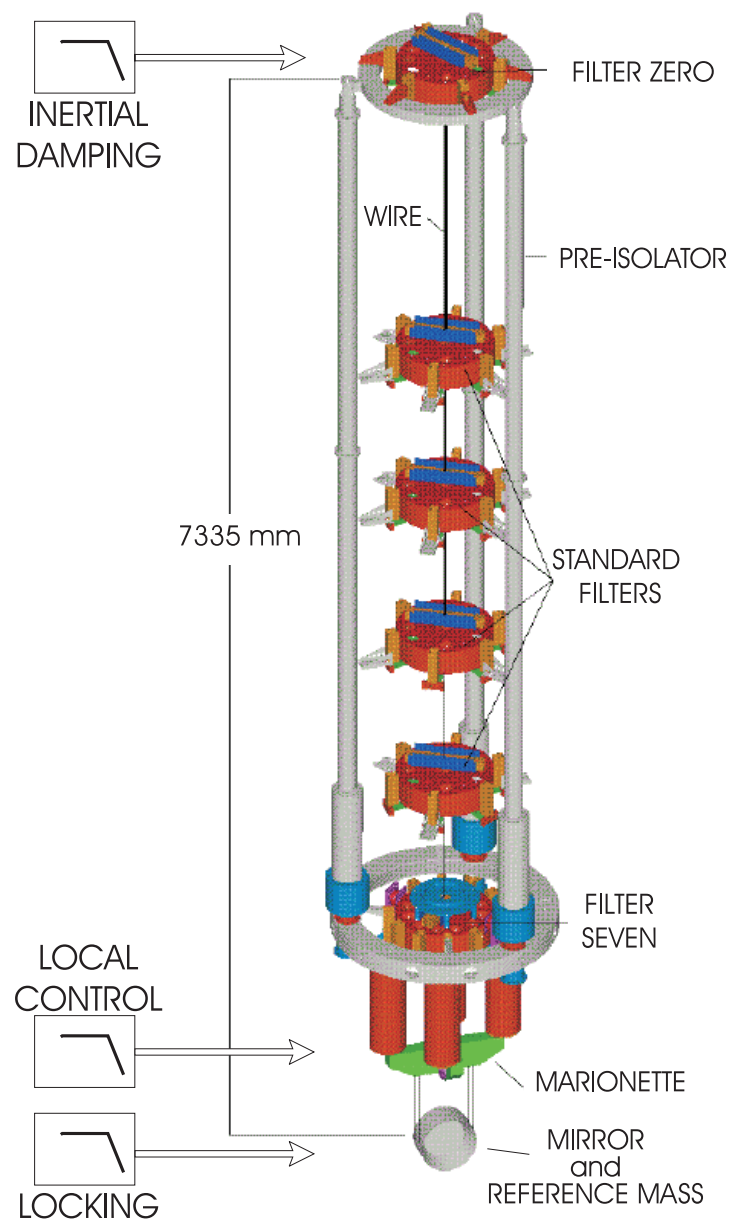

Figure 4. Schem e of the Virgo Superattenuator chain: in the CTF the feedback is exerted at three stages: inertial damping is perform ed at the inverted pendulum stage, local control through them arionette, and the interferom eter locking keeping force acts on them inorthrough the reference $m$ ass.

\section{The com $\mathrm{m}$ issioning of the $3 \mathrm{~km}$ long V IR G O}

In order to test the stability and robustness of all of the sub-system s involved in the operations and to get.som e experience in designing the various controlsystem $s$, the $V$ irgo com $m$ issioning activity has been organized in steps of increasing com plexity: the separate com $m$ issioning of the $\mathrm{N}$ orth and $\mathrm{W}$ est Fabry-Perot cavities, follow ed by the com $\mathrm{m}$ issioning of the recom bined $\mathrm{M}$ ichelson Fabry-Perot TFF, and eventually the com $\mathrm{m}$ issioning of the recycled M ichelson Fabry-Perot ITF. Shortperiods of continuous data-taking (the so-called com m issioning runs) have taken place every two to three m onths since Novem ber 2003, in order to check the evolution of the detector and the consequent progress in the level of sensitivity. Five com m issioning runs have been perform ed so far:

- C1 - N orth cavity longitudinally controlled (14-17 N ovem ber2003);

- C2 - N orth cavity longitudinally controlled, plus autom atic alignm ent (20-23 February 2004); 
- C3 - Two configurations: North cavity as in C2 plus the frequency stabilisation servo (23-26 A pril 2004); first data-taking $w$ ith the ITF locked in recom bined m ode (26-27 A pril 2004);

- C4 - ITF longitudinally controlled in recom bined m ode, w ith suspension tidal control, autom atic alignm enton both the arm s, frequency stabilization servo (24-29 June 2004);

- C5 - Tw o configurations: TFF in recom bined m ode as in C4, plus end suspensions w ith full hierarchical control (2-6 D ecem ber 2004); first data-taking w ith the TF locked in recycled m ode (6-7 D ecem ber 2004);

The com $\mathrm{m}$ issioning of a single arm was conchuded $\mathrm{w}$ ith $\mathrm{C} 3, \mathrm{w}$ ith an autom atic alignm ent and a frequency servo running for this configuration. The evolution of the detectors w olking in recom bined and in recycled m ode w ill be described in sections 42 and 4.5, where the attention $w$ ill focus upon the tw o m ost recentdata-taking during $\mathrm{C} 4$ and $\mathrm{C} 5$.

\section{1. The longitudinalcontrol}

The nom inal sensitivity of an interferom etric detector such as V irgo is achieved by selecting an appropriate w orking point, w ith laser light resonant in the optical cavities, and the output port tuned on the dark fringe. These conditions translate into fixed relationships betw een the laser lightw avelength and four independent lengths of the ITF [20]:

- the length of the recycling cavity (PRCL),

- the differential length of the shortM ichelson arm S (M ICH ), $l_{1}-l_{2}$;

- the com m on (CARM) and the differential (DARM) length of the two long arm $\mathrm{s}_{1} \mathrm{~L}_{1}+\mathrm{L}_{2}$ and $\mathrm{L}_{1}-\mathrm{L}_{2}$.

W hile the expected sensitivity is of the order of $10^{-18} \mathrm{~m} / \sqrt{\mathrm{H} \mathrm{z}}$, the allow ed deviation from the w orking point is $10^{-12} \mathrm{~m}$ m s. A feedback controlsystem is needed to keep the ITF locked on the required interference conditions. Relative displacem entof the m inors is detected using a camier beam phase m odulated at $6 \mathrm{M} \mathrm{H} \mathrm{z}$. U sing a standard PD H technique all the lengths involved can be reconstructed by m ixing the signals produced by the photodiodes, which are placed at different output ports of the $\mathrm{TF}$. These enor signals are digitized and sent to the V irgo global control system (G lobal Control [20]), w hich com putes the comections to be applied to the m inors by the actuators at the level of the reference $m$ ass.

A localcontrolsystem, referred to the ground, is active in the bottom partof each SA in order to keep the longitudinal displacem entof the m inors below 1 m m $\mathrm{m}$. This also keeps the local angularm otion of the $\mathrm{m}$ inors below $1 \mu \mathrm{rad} \mathrm{m}$ s and allow s the acquisition of the longitudinal lock of the interferom eterusing a lim ited actuation force, thus preventing noise reintroduction in the detection band.

\section{2. The recom bined interferom eter}

A s an interm ediate step tow ards the full configuration, the interferom eterw as com $m$ issioned in recom bined m ode for a large part of 2004. In this m ode the optical schem e differs from 
the final configuration in that the PR $\mathrm{m}$ inor is significantly $\mathrm{m}$ isaligned, so that only three lengths instead of four have to be longitudinally controlled: CARM ,DARM and M ICH .U sing the end transm itted signals the tw o long arm s can be controlled independently, acting on the comesponding end $\mathrm{m}$ inors. A s soon as the cavities are locked, $\mathrm{M}$ ICH is controlled $\mathrm{w}$ ith the outputportdem odulated signal (oraltematively the reflected dem odulated signal) filtered and sent to the B S . By applying this strategy the lock is usually acquired in a few seconds.

Because of the low pow er upon the end photodiodes, the transm itted signals are electronic noise-lim ited: once the lock is acquired, they have to be replaced by another set of less noisy signals. In a steady state CARM is also attained using by the in-phase dem odulated com ponent of the light reflected by the TFF. Instead, DARM is attained by the in-phase outputport light, which is slightly contam inated by M ICH , m ainly provided by the otherquadrature of the ITF reflected light. L inearcom binations of the enor signals are com puted to provide the conrection forces to the m inors. This phase is called linear locking.

\section{3. The Comm issioning Run C 4}

During $\mathrm{C} 4$, the interferom eter was operated for five days in recom bined m ode. The longitudinal degrees of freedom were locked according to the linear locking schem $e$ previously described, w ith the autom atic alignm entrunning on both arm sand suspension tidal control on the end $\mathrm{m}$ inors. The laser frequency w as actively stabilized on CARM , w hich w as locked on the reference cavity in line w ith the frequency servo strategy developed in V irgo, the so-called Second Stage of Frequency stabilization. The O M C w as locked on the dark fringe, so thatD ARM could be controlled by the filtered outputdem odulated signal. The interferom etric schem e in the data-taking m ode is described in figure 5. The longest continuous lock during C 4 w as about 28 hours. A 119 lock losses thatoccumed w ere analyzed and understood. A t the beginning of the run som e acoustic noise injection w as perform ed in the laser and detection laboratory, to study the possible couplings $w$ ith the dark fringe signal [21]. A softw are and hardw are injection of inspiral events $w$ as also perform ed [23] during the data-taking, to test som e of the elem ents of the analysis chain and to characterize the detector stability during the run.

At the beginning of the run som e calibration noise injection was perform ed in order to produce the sensitivity curve. The result is plotted in figure 6 , together $w$ ith the $m$ ain noise contributions. The sensitivity is still lim ited by controlnoise at low frequency, by the m inor actuatornoise in the interm ediate frequency range and by laser frequency noise, starting from som e hundreds of $\mathrm{H} z$.

\subsection{Reduction of actuation noise}

Longitudinal control during $\mathrm{C} 4 \mathrm{w}$ as acquired and m aintained by acting on the minor at the level of the reference $\mathrm{m}$ ass. The noise injected by the recoil $\mathrm{m}$ ass actuators into the interferom eter is a severe lim it to the sensitivity of V irgo: at $20 \mathrm{H} \mathrm{z}$ it is m ore than 1000 tim es larger than the design sensitivity. It is m ainly contributed to by the 16 bitDAC noise (300 $\mathrm{nV} /$ squt $(\mathrm{H} \mathrm{z})$ ) and the coildrivernoise (70 nV/squt(H z)) and is converted into equivalentm inror 


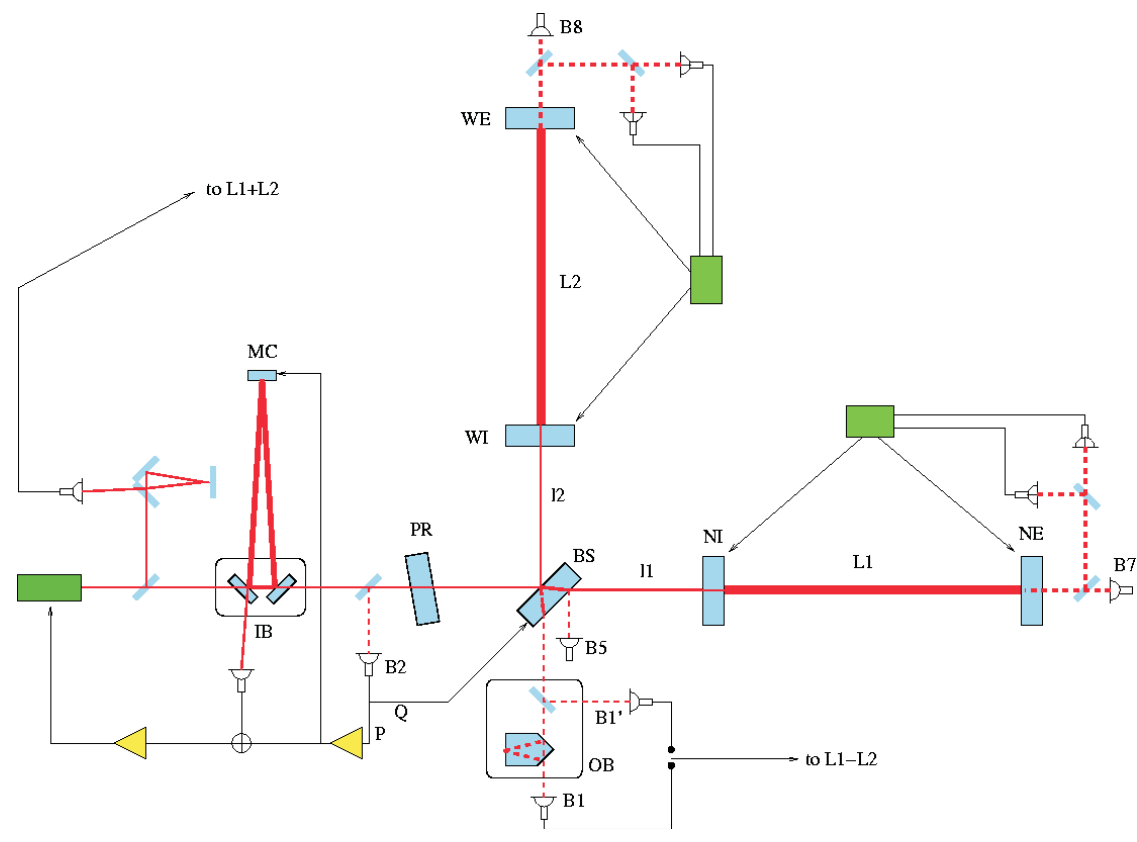

Figure 5. C4 configuration: the data-taking $m$ ode consisted of the TF locked in recom bined m ode according to the linear locking schem $e, w$ ith the autom atic alignm ent running on both arm s. In this state, the frequency stabilization control system is engaged. W ith the laser frequency pre-stabilized on the $\mathbb{I M} C$, the CARM locking loop is sw itched off and the comesponding PD H enor signal is added into the emorpoint of the $\mathbb{I M} C$-loop bandw idth 1 $\mathrm{kH} z$ ). The sam e signal is applied to the length of the $\mathbb{M} \mathrm{C}, w$ ith a bandw idth of about 200 $\mathrm{H} \mathrm{z}$ : in this w ay the laser frequency and the length of the $\mathbb{I M} C$ are stabilized on CARM, which provides a better frequency stability at frequencies higher than the internal resonances of the SA . The low frequency stabilization is achieved locking CARM on the RFC length.

displacem ent by a reasonably large coupling factor: $130 \mathrm{~m}$ icrons $N$. Such a large coupling factor has been adopted in order to ease the lock acquisition. O nce the lock is acquired, the residual force to be exerted is largely in the low frequency region (D C -5 H z), where tidal drifts and resonant m otion have to be com pensated, and very sm all elsew here. Therefore, the gain of the coil driver (and the comesponding noise) cannot be reduced, unless a large fraction of the low frequency force is reallocated to the upper stages. This is in fact the Virgo suspension hierarchical control strategy: once the lock is acquired, the locking force is split over three actuation stages in a hierarchical w ay. The comection in the range D C $0.01 \mathrm{H} \mathrm{z}$, that com pensates for earth tides, is reallocated upon the soft inverted pendulum ; the force in the range $0.01-8 \mathrm{H} \mathrm{z}$, where all the suspension resonances fall, is reallocated to the $m$ arionette. Consequently, the residual force on the reference $m$ ass is strongly reduced, and a strong reduction of the coildrivergain becom es possible.

$\mathrm{W}$ ith respect to the reference $\mathrm{m}$ ass-m inor system, the upper actuation stage reveals m ore com plex dynam ics. There is an intrinsic and non negligible coupling betw een the horizontal actuators pushing on the $\mathrm{m}$ arionette and the pitch $\mathrm{m}$ otion induced on the $\mathrm{m}$ inor. Therefore, the locking of the ITF from the m arionette, requires the use of all fouractuators available w ith a proper frequency dependent diagonalization. 


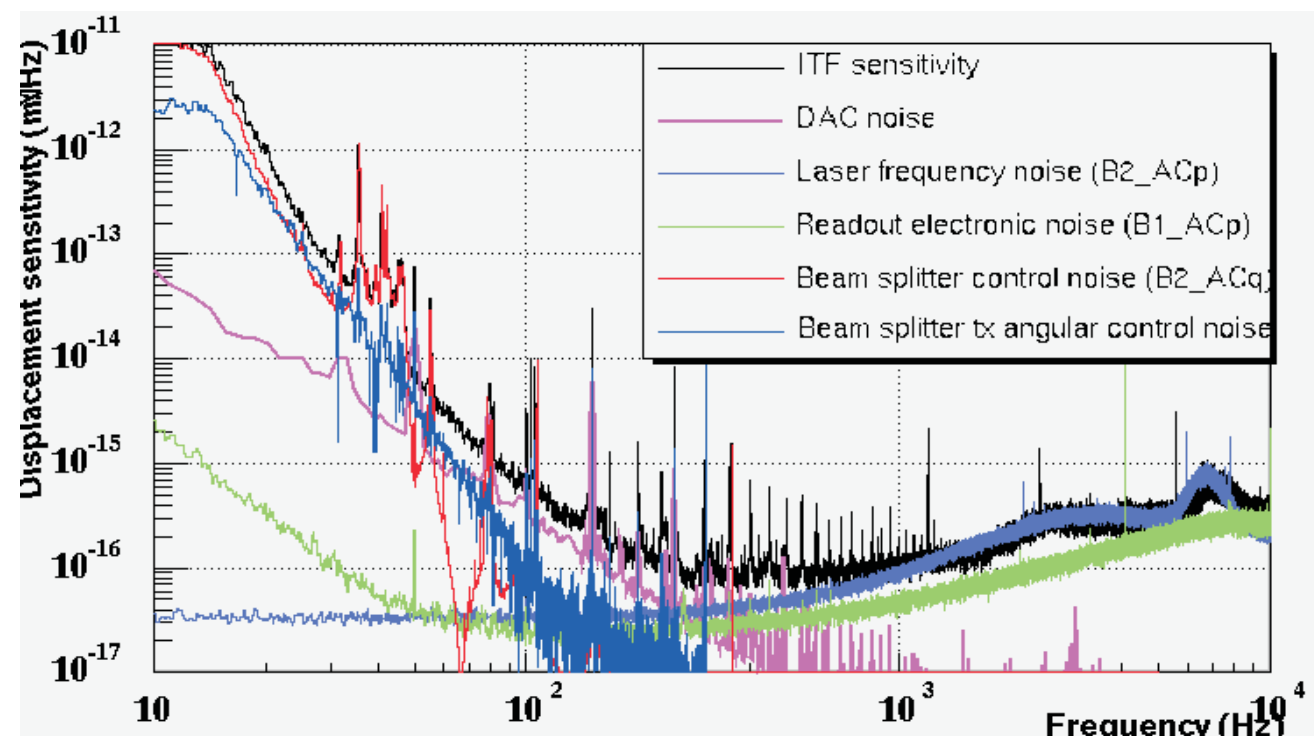

Figure 6. Sensitivity curve in $\mathrm{C} 4$ expressed in $\mathrm{m} / \sqrt{\mathrm{Hz}}$, $\mathrm{w}$ ith the $\mathrm{m}$ ain noise contributions: control noise at low frequency, m inor actuator noise (essentially 16 bit DAC noise) in the interm ediate frequency range and laser frequency noise starting from som e hundreds of $\mathrm{H}$.

O nce the lock is acquired using the recoil m ass only, the recom bined ITF should be fully locked before reallocating the force to the upper stage: it is necessary that second stage of frequency stabilization is engaged, otherw ise the frequency noise w ould cause saturation of the $m$ arionette actuators. A fter reallocation, the reference $m$ ass residual conrection alw ays rem ains below $10 \mathrm{~m} \mathrm{~V}$, allow ing a reduction of the coil driver am plification by a factor of 1000 .

The $\mathbb{T F} \mathrm{F}$ as running in recom bined m ode during the firstpart of $\mathrm{C} 5$, w hen a full hierarchical control of the end suspensions $w$ as successfully tested.

\subsection{The lock acquisition of the recycled ITF}

A s V irgo and L IG O have sim ilaropticalset-ups, the lock acquisition strategy developed [24] and adopted in the LIGO interferom eter [25] was taken as a starting point for the lock acquisition schem e of the Virgo recycled interferom eter. This baseline technique consists in sequentially controlling the four degrees of freedom of the ITF, dynam ically changing the optical sensing $m$ atrix to com pensate the variation of the fields in the course of lock acquisition. Som e interm ediate stable states w ere locked applying the L IG O strategy, and som e full lock acquisition trials w ere perform ed. A t the sam e tim e an altemative technique w as developed: the first tests rapidly provided prom ising results, and experim ental activity on the baseline technique w as subsequently intemupted. 


\subsection{Lock acquisition}

The basic idea of the new lock acquisition technique is that the TFF is locked outside the w orking point for the dark fringe. In this w ay a good fraction of light escapes through the outputportand the pow erbuild-up in the recycling cavity is low. Then the ITF is adiabatically broughton to the dark fringe. This technique is referred to as variable finesse [22], because the finesse of the recycling cavity changes during the lock acquisition path. The procedure starts $\mathrm{w}$ ith the PR $\mathrm{m}$ inror initially $\mathrm{m}$ isaligned by som e urads. The sim ple M ichelson is controlled on the half fringe, using the output port D C signal, while the two arm s are independently locked using the end photodiodes, as in the recom bined configuration. The pow er recycling cavity length is controlled using the reflected 3 f-dem odulated signal. In this w ay all the four longitudinal degrees of freedom of the $\mathrm{TF}$ are locked in a stable w ay from the beginning of the lock acquisition procedure, preventing excitation of the m inors. From this starting condition the $\mathrm{PR}$ is realigned, w hile alw ays $m$ aintaining the $\mathrm{M}$ ichelson on the half fringe, giving a very low recycling gain.

In order to increase the recycling gain the M ichelson has to be broughton to the dark fringe: this is done adiabatically, decreasing the offset in the M ichelson enror signal. A the sam e tim e, the control schem e changes. The end photodiodes can only be used to independently control the cavities w hen the IF is farfirom the dark fringe: $w$ hen nearing the dark fringe they begin to couple strongly and a com m on and differential control has to be activated to keep the lock. Then a frequency stabilization servo is engaged, controlling CARM w ith a very high bandw idth: consequently, the contam ination by this degree of freedom on all the photodiodes is cancelled. DARM is kept in a locked state by one of the end photodiode signals. The final step consists of sw itching from the D C to a dem odulated signal to control the M ichelson length. Eventually the offset in the M ichelson enror signal is rem oved, the TrF goes on to the dark fringe and the recycling cavity gain increases up to the m axim um value.

A pplying this technique the lock acquisition of the full V ingo IFF w as reached for the first tim e on 26th O ctober 2004, and tested in the latter part of C 5. A typical lock acquisition sequence takes few $m$ inutes and it provides a determ inistic and repeatable lock. The final recycling cavity gain w as m easured to be around 25 .

\subsection{Sensitivity progress}

The $3 \mathrm{~km}$ Virgo detector has been in com $\mathrm{m}$ issioning for about one and half years. The first lock of a single Fabry-Perot arm w as realized in O ctober 2003: after exactly one year, the lock of the recycled ITF w as perform ed (see figure 7). In betw een, the com $\mathrm{m}$ issioning of the recom bined $\mathrm{TF} \mathrm{F}$ w as also realized, $\mathrm{w}$ ith the continous im provem entof the various sub-system s and controls involved in the operations: longitudinal lock, autom atic alignm ent, frequency stabilization servo, full hierarchicalsuspension control.

D uring this one-year period the displacem ent sensitivity of the detector has evolved from $10^{-11} \mathrm{~m} / \sqrt{\mathrm{H}} \mathrm{z}$ to less than $10^{-16} \mathrm{~m} / \sqrt{\mathrm{H}}$. 


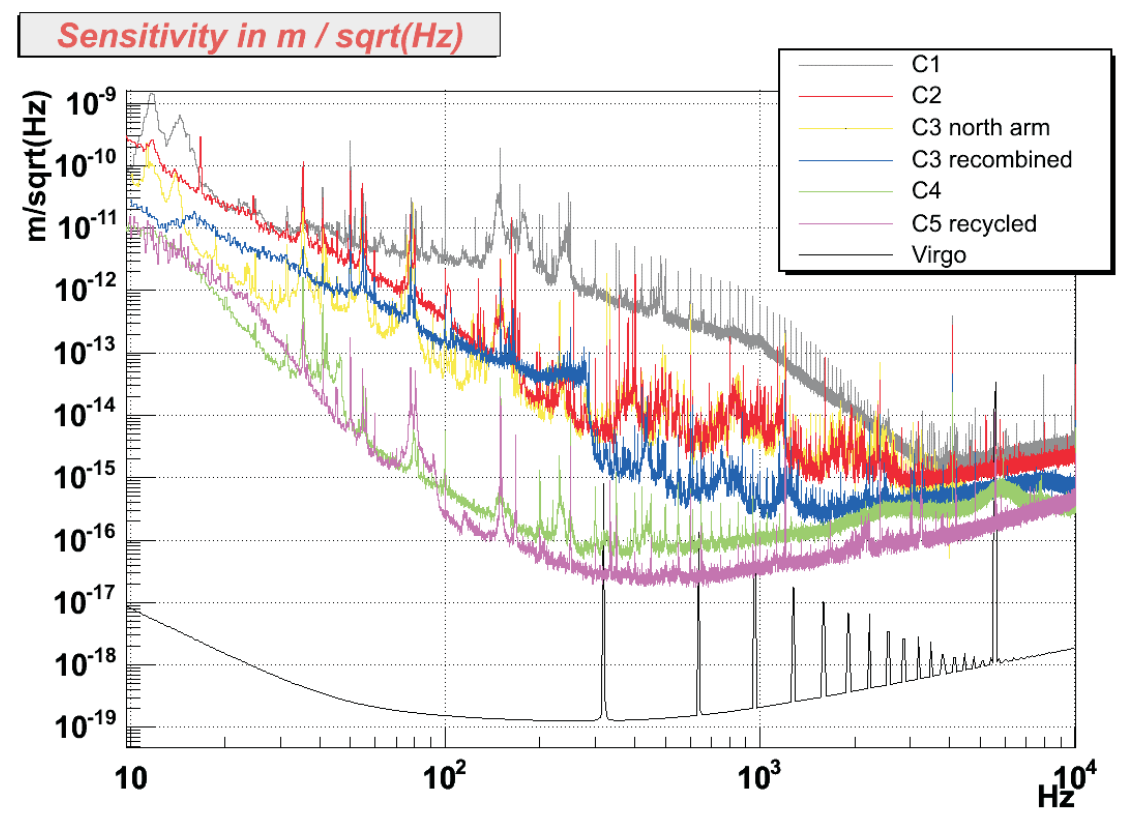

Figure 7. Progress in the $V$ irgo sensitivity in approxim ately one year of com $m$ issioning. The fact that during the sum m er of 2004 a laser pow er attenuator w as installed in the injection system also need to be considered: in $\mathrm{C} 5$ the laser light entering the $\mathrm{TF} \mathrm{F}$ w as around $0.7 \mathrm{~W}$, instead of $7 \mathrm{~W}$ as in the previous runs.

\section{Conchision}

The gravitational w ave intenferom eters are the extrem e evolution of the classical M ichelson interferom eter. Even if their goal is the m easurem ent, at the typical frequency of $100 \mathrm{H} \mathrm{z}$, of spectral densities of relative displacem ents, rather than static lengths, the order of m agnitude of the targetsensitivity (m uch less than the diam eterof an atom ic nucleus) sounds astounding. The progress in the sensitivity of the $\mathrm{V}$ irgo intenferom eter tow ards the target sensitivity is a good dem onstration of the w ay in w hich this m easurem ents can be attained. A fter one year of upgrades, the displacem ent sensitivity of the detector has evolved from $10^{-11} \mathrm{~m} / \sqrt{\mathrm{H}}$ to less than $10^{-16} \mathrm{~m} / \sqrt{\mathrm{H} z}$. Presently, the com $\mathrm{m}$ issioning of the recycled $\mathbb{T} \mathrm{F}$ is on-going, $\mathrm{w}$ ith the goal to im prove the robustness of the longitudinal lock, at the sam e tim e putting into operation otherm ain controlsystem successfully im plem ented in the recom bined configuration and reducing extemal noise sources. The characterization of the noise contributions to the sensitivity constitutes another fundam ental task, w ith the prospect of a science nun, close to the target sensititivy, before the end of 2005.

$R$ eferences

[1] A A M ichelson and E IV M orley, PhilosM ag S 5, 24 (151), 449-463 (1887).

[2] F.A cemese etal., Status ofVirgo, C lass Q uantG rav., 21, 385-393 (2004). 
[3] D .Sigg etal., Com m issioning of the LIG O detectors,C lass.Q uant.G rav., 19 (7) , 1429-1435 (2002).

[4] M A ndo etal., Current status ofTAM A, C lass.Q uant.G rav. 19 (7) , 1409-1419, (2002).

[5] B.W i]lke etal., The G EO 600 gravitational wave detector, C lass.Q uant.G rav., 19 (7), 1377-1387 (2002).

[6] M E.Gertsenshtein, V I. Pustovoit, Soviet Physics, JETP , 16, 433, (1962).

[7] R .V Pound, Electronic Frequency Stabilization of M icrow ave O scillators, R ev. Sci. Instrum . 17 490-505 (1946)

[8] R .N PD rever et al, Laser phase and frequency stabilization using an optical resonator, A ppl. Phys. B : Photophys. LaserChem . 31 (1983) 97-105

[9] L . Schnupp, The European Collaboration M eeting on Interferom etric D etection of G ravitational W aves, 37,66, 92, (Somento, 1998).

[10] M onrison E ., M eers B J., Robertson D I., W ard H ., Autom atic alignm ent of optical interferom eters, A pplo pt., 33, 5041 (1994).

[11] D . Z .A nderson, A lignm ent of resonantoptical cavities, A ppl. O pt. 23 2944-2949 (1984).

[12] D . Babusci, H . Fang, G . G iordano, G . M atone, L . M atone, V. Sannibale, Alignm ent procedure for the VIRG O interferom eter: experim entalresults from the Frascatiprototype, Phys.LettA 226,31-40 (1997).

[13] L . Pinard etal., O ptical System s design 2003 proc., 2003.

[14] JM M ackow skietal., O pt.Q uantElectr., 31 (5/7), 507-514, (1999).

[15] G B allardin etal., M easurem entof the VIRG O superattenuatorperform ance for seism ic noise suppression, Rev.Sci. Instrum . 72 3643-3652 (2001).

[16] G Losurdo et al., An inverted penduhum pre-isolator stage for the VIRGO suspension system , Rev. Sci. Instrum ., 70 (5), 2507-2515 (1999).

[17] G .B allardin etal, M easurem entof the transfer function of the steering filter of the V IRG $O$ superattenuator suspension, Rev.Sci. Instrum ., 72, 3635 (2001).

[18] M .Bemardinietal, Suspension laststage for the m inors of the V IRG O interferom etric gravitational w ave antenna, Rev.Sci. Instrum ., 70, 3463 (1999).

[19] G .Losurdo etal, Inertial control of the $m$ inror suspensions of the VIRG O interferom eter for gravitational wave detection, Rev. Sci. Instrum ., 72, 3654-3661 (2001).

[20] F.C avalier, Le controle global de Virgo, These $d$ ' $\mathrm{H}$ abilitation a diriger des R echerches, U niversitè de Paris Sud, LA L 01-69 (2001).

[21] I. Fiori, A first,study of environm entalnoise coupling to the Virgo interferom eter, these proceedings.

[22] L . B arsottietal, Status of Virgo, C lass. and Q uant. G rav., proc.GW DAW 2004 ., to be published.

[23] L B .Bosi, Inspiral analysis of the Virgo com m issioning nun 4, these proceedings.

[24] M Evans, Lock acquisition in ResonantO ptical Interferom eters, PhD Thesis, CA LTECH (2001)

[25] M Evans etal., Lock acquisition of a gravitational-w ave interferom eter, O PT ICS LETTERS vol27, n.8. April 15, (2002)

[26] K A rai and the TAM A collaboration Sensing and controls for pow er-recycling ofTAM A 300, subm itted to Class. Q uantum G rav. 